\title{
Endoscopic treatment for bleeding peptic ulcers: randomised comparison of adrenaline injection and adrenaline injection $+\mathrm{Nd}$ :YAG laser photocoagulation
}

\author{
L A Loizou, S G Bown
}

\begin{abstract}
Forty two patients with haemorrhage from peptic ulcers with visible vessels were enrolled in a randomised study comparing endoscopic haemostasis with adrenaline (1:10 000) injections (adrenaline group) and adrenaline injection+neodymium yttrium-aluminium-garnet (Nd:YAG) laser photocoagulation (adrenaline +laser group). The two groups (21 patients each) were well matched for factors affecting outcome. Surgery was performed for continued haemorrhage uncontrolled by endoscopic treatment or rebleeding after two endoscopic treatments. Haemostasis after one treatment was similar in the two groups: adrenaline $16 / 21(76 \%)$, adrenaline+laser $18 / 21(86 \%)$. Haemostasis after two treatments was numerically $(0.05<\mathrm{p}<0.10)$ greater in the adrenaline+laser group: $21 / 21(100 \%) v 18 / 21$ $(86 \%)$. Three patients $(14 \%)$ in the adrenaline group underwent uneventful emergency surgery. There were no deaths or procedure related complications in either group. Most bleeds from peptic ulcers with visible vessels can be controlled endoscopically without the need for surgery. Both treatments in this study proved highly efficacious in securing haemostasis. Adrenaline injection treatment seems to be the treatment of choice in view of its simplicity, low cost, and availability. Additional Nd:YAG laser treatment may provide a marginal improvement in efficacy, although a much larger trial would be required to prove this.
\end{abstract}

Mortality from peptic ulcer haemorrhage occurs almost exclusively in patients older than 60 years as a result of the postoperative complications of surgery performed for continued or recurrent bleeding. ${ }^{1}$ Further haemorrhage after hospital admission is associated with a seven to 10 -fold increase in mortality. ${ }^{23}$ In an attempt to make an impact on mortality from peptic ulcer haemorrhage, several endoscopic haemostatic modalities have been developed over the past decade to prevent further bleeding and thus obviate the need for high risk emergency surgery. Such treatment is targetted for ulcers with a bleeding or non-bleeding 'visible vessel' as these are the only endoscopic stigmata of haemorrhage associated with a high risk of further bleeding. ${ }^{46}$ The risk of further haemorrhage is $75-80 \%$ if active bleeding is seen and $40-60 \%$ for non-bleeding visible vessels. ${ }^{56}$ Well designed randomised controlled trials of neodymium yttrium-aluminium- garnet (Nd:YAG) laser photocoagulation in bleeding peptic ulcers have shown an appreciable reduction in rebleeding rate, need for emergency surgery, and mortality in some of the studies. $^{6-9}$ As preliminary clinical and experimental evidence suggested that injection of the bleeding point with adrenaline (1:10000 dilution) before laser photocoagulation improved both the efficacy and safety of laser treatment, ${ }^{10}$ we have been using this combined haemostatic treatment at this hospital since early 1988. Laser haemostasis, although efficacious, has been criticised because of cost and non-portability of the equipment and the high level of operator expertise required. In contrast, endoscopic adrenaline injection as primary haemostatic treatment is cheap and relatively easy to perform and was shown in a recent randomised trial to reduce appreciably further haemorrhage and need for emergency surgery compared with conservative management in patients with bleeding peptic ulcers. " We therefore decided to perform a randomised comparison of endoscopic adrenaline injection alone and adrenaline injection + Nd:YAG laser photocoagulation of bleeding peptic ulcers to discover whether combined treatment confers any advantage over the simpler alternative of injection monotherapy.

As a previous randomised comparison of Nd:YAG laser versus no endoscopic treatment conducted in this hospital showed a significantly lower mortality in laser treated patients, ${ }^{6}$ it was not thought ethical to include a control group in the present trial.

\section{Patients and methods}

PATIENTS, INCLUSION CRITERIA, AND RANDOMISATION

All patients admitted to this hospital with haematemesis or melaena, or both, between 1 July 1988 and 19 November 1989 were considered for inclusion in the trial. Upper gastrointestinal endoscopy was performed within 18 hours of admission; patients with clinical shock or massive bleeding at presentation were endoscoped soon after initial haemodynamic resuscitation. Most of the diagnostic endoscopies and all haemostatic treatments were performed by one operator (LAL) and the trial was concluded when his research fellowship finished.

Patients were included in the trial, with their informed consent, if a gastric, duodenal, or stomal ulcer with a bleeding or non-bleeding 'visible vessel' in its base accessible to haemo- 
static treatment was found at endoscopy. Such ulcers carry the highest risk of further haemorrhage and hence mortality; ulcers with minor or no stigmata of recent haemorrhage were excluded as the probability of rebleeding is minimal. ${ }^{+6} \mathrm{~A}$ non-bleeding visible vessel was defined as a raised red or blue spot resistant to washing. ${ }^{5}$ To facilitate examination of the ulcer crater for the presence of stigmata of recent haemorrhage, secretions and adherent blood clots were washed off. No patients were excluded on the basis of large vessel size or multiplicity of ulcers, if in the latter case one ulcer could be positively identified as the bleeding source.

Patients were stratified into two groups according to whether the visible vessel was actively bleeding at the time of endoscopy or not and separately randomised, by opening sealed numbered envelopes, to receive either adrenaline injection treatment or adrenaline injection + Nd:YAG laser photocoagulation.

\section{ENDOSCOPIC TECHNIQUES}

Endoscopic adrenaline injections were performed with a flexible injector, $0.7 \mathrm{~mm}$ diameter needle (Diagmed, UK), passed through the working channel of a GIF 1T20 Olympus UGI endoscope. Two $\mathrm{ml}$ aliquots of 1:10000 adrenaline were injected in the ulcer base in a circumferential fashion as close to the visible vessel as possible; direct injection of the vessel itself was not performed. Non-bleeding visible vessels were injected with $10-12 \mathrm{ml}$ of adrenaline. When there was active bleeding larger volumes of adrenaline (up to $30 \mathrm{ml}$ ) were injected in an attempt to obtain haemostasis.

In patients randomised to receive combined injection and laser treatment adrenaline preinjection of the vessel was followed by photocoagulation using the Nd:YAG laser (Flexilase, Living Technology, Glasgow) which can generate a continuous wave emission of $1064 \mathrm{~nm}$ (near infra red) and a power output of $1-100 \mathrm{~W}$. Laser light is transmitted through a $600 \mu \mathrm{m}$ quartz fibre enclosed within a teflon catheter (2 $\mathrm{mm}$ diameter) which is passed down the working channel of an endoscope fitted with a safety filter in its eyepiece. A coaxial stream of carbon dioxide or air is delivered through the teflon catheter to cool the fibre tip and also clear the target of blood and debris. The tip of the fibre is positioned approximately $10 \mathrm{~mm}$ away from the visible vessel and multiple (on average 6-8) $75 \mathrm{~W}, 0.5 \mathrm{~s}$ shots of laser energy are applied in a tight ring as close to the bleeding point as possible but avoiding a direct hit.

\section{MONITORING AND FURTHER MANAGEMENT}

Patients with continuing spurting (arterial) haemorrhage despite two attempts to secure haemostasis with endoscopic treatment underwent emergency surgery after further haemodynamic resuscitation. After successful haemostatic treatment patients were transferred to medical wards for observation. All were treated with oral ranitidine $300 \mathrm{mg}$ twice daily (reducing to $300 \mathrm{mg}$ once daily after one week) and received blood transfusions as indicated by haematological and clinical parameters. The attending physicians or surgeons were made aware of the exact endoscopic findings and treatment given in each case. A definite diagnosis of rebleeding was made if there was: (a) fresh haematemesis; (b) fresh melaena accompanied by clinical shock (blood pressure $<100 \mathrm{~mm} \mathrm{Hg}$, heart rate $>100 / \mathrm{min}$ ) or falling haemoglobin despite further blood transfusion; (c) fresh blood in the upper gastrointestinal tract at repeat endoscopy.

All first rebleeds were treated by a second session of endoscopic haemostatic treatment according to the initial randomisation. Emergency surgery was undertaken only after a second documented episode of rebleeding.

TRIAL END POINTS AND STATISTICAL METHODS Patient involvement in the trial extended up to the time of death or discharge from hospital. The two treatment groups were compared for final haemostasis rate, need for emergency surgery, mortality, hospitalisation time, and transfusion requirements. Proportional differences in the two groups were tested for significance by the $\chi^{2}$ test with Yates's continuity correction. Continuous (integral) data were compared using the unpaired, two sided Wilcoxon rank sum test. Significance was set at the $5 \%$ level $(\mathrm{p}<0 \cdot 05)$.

\section{Results}

During the study period 42 patients presenting with haematemesis or melaena, or both, were found at endoscopy to have peptic ulcers with bleeding or non-bleeding visible vessels. One patient, who was not randomised as the trial endoscopist was unavailable, had a duodenal ulcer with a non-bleeding visible vessel which was treated by adrenaline injections and did not rebleed. No patients were excluded on the basis of inaccessibility or severity of bleeding.

Of the 42 patients recruited to the trial, 21 were randomised to receive adrenaline injection treatment alone and the other 21 adrenaline injection $+\mathrm{Nd}$ :YAG laser photocoagulation. Patient details are shown in Table I. The two treatment groups were well matched for factors known to affect prognosis. Only $18 \%$ of patients in the adrenaline group and $27 \%$ in the adrenaline $+\mathrm{Nd}$ :YAG laser group reported dyspeptic symptoms in the two weeks preceding the index bleed. Thirty per cent of patients in the former group and $43 \%$ in the latter were taking non-steroidal antiinflammatory drugs. Nine patients $(22 \%)$ were referred to this hospital for endoscopic haemostasis from outside centres as they had rebled, often repeatedly, after admission and were considered unfit for emergency surgery.

Haemostasis rates are shown in Table II. Both in patients with bleeding and non-bleeding visible vessels, initial haemostasis, rebleeding, and final haemostasis rates were similar in both groups; numerical differences were not significant. It should be noted that a second session of adrenaline injection or adrenaline injection + Nd:YAG laser treatment secured final haemostasis in five of six patients $(83 \%)$ who rebled 
TABLE I Details of patients recruited to the study

\begin{tabular}{|c|c|c|}
\hline & $\begin{array}{l}\text { Adrenaline } \\
(n=21)\end{array}$ & $\begin{array}{l}\text { Adrenaline } \\
+ \text { laser } \\
(n=21)\end{array}$ \\
\hline Mean (SD) age (years) & $61(17)$ & $60(18)$ \\
\hline Males:females & $15: 6$ & $16: 5$ \\
\hline No $(\%)$ with concomitant disease & $9(43)$ & $8(38)$ \\
\hline \multicolumn{3}{|c|}{ No $(\%)$ in clinical shock (blood pressure } \\
\hline$<100 \mathrm{~mm} \mathrm{Hg})$ & $10(48)$ & $12(57)$ \\
\hline Mean (SD) initial haemoglobin $(\mathrm{g} / \mathrm{l})$ & $98(23)$ & $95(26)$ \\
\hline \multicolumn{3}{|l|}{ Mean (SD) initial blood transfusion } \\
\hline requirement (units) & $4 \cdot 3(3 \cdot 1)$ & $4 \cdot 7(2 \cdot 6)$ \\
\hline Gastric:duodenal:stomal ulcers & $10: 10: 1$ & $8: 12: 1$ \\
\hline Bleeding:non-bleeding visible vessels & $3: 18$ & $5: 16$ \\
\hline
\end{tabular}

after the first session of endoscopic treatment. Of the nine patients referred from outside centres, final haemostasis was achieved endoscopically in all but one, randomised to the adrenaline injection group, who was bleeding massively at the time of endoscopy from the posterior duodenum. She underwent emergency underrunning of the gastroduodenal artery plus vagotomy and pyloroplasty and made an uneventful recovery.

The need for emergency surgery, mortality, total blood transfusion requirement, and hospitalisation time in the two groups is shown in Table III. The numerical difference in the emergency surgery rate for further haemorrhage between the two treatment groups was not significant $(0.05<\mathrm{p}<0 \cdot 1)$. The implementation of a policy of endoscopic haemostasis for peptic ulcer haemorrhage using adrenaline injection or adrenaline injection $+\mathrm{Nd}$ :YAG laser treatment, with repeat treatment for first rebleeds, resulted in an overall emergency surgery rate of $7 \%$ and a $0 \%$ mortality. The average hospitalisation time was less than 10 days in both groups.

Active bleeding from the visible vessel was precipitated during laser treatment in two $(9 \cdot 5 \%)$ patients; haemorrhage was arrested by repeat adrenaline injection and further laser coagulation in both patients. In the adrenaline injection group treatment provoked spurting haemorrhage in one patient with a high posterior wall

TABLE II Haemostasis rates (percentages in parentheses)

\begin{tabular}{|c|c|c|}
\hline & Adrenaline & $\begin{array}{l}\text { Adrenaline } \\
\text { + laser }\end{array}$ \\
\hline \multicolumn{3}{|l|}{ Bleeding visible vessels: } \\
\hline Initial haemostasis & $2 / 3(67)$ & $5 / 5(100)$ \\
\hline Rebleed & $0 / 2$ & \\
\hline Successful second treatment & & $2 / 2$ \\
\hline Final haemostasis & $2 / 3(67)$ & $5 / 5(100)$ \\
\hline \multicolumn{3}{|l|}{ Non-bleeding visible vessels: } \\
\hline Initial haemostasis & $17 / 18(94)$ & $16 / 16(100)$ \\
\hline Rebleed & $3 / 17(18)$ & $1 / 16(6)$ \\
\hline Successful second treatment & $2 / 3$ & $1 / 1$ \\
\hline Final haemostasis & $16 / 18(89)$ & $16 / 16(100)$ \\
\hline \multicolumn{3}{|l|}{ Total } \\
\hline Haemostasis after one treatment & $16 / 21(76)$ & $18 / 21(86)$ \\
\hline Haemostasis after two treatments & $18 / 21(86)$ & $21 / 21(100)$ \\
\hline
\end{tabular}

TABLE III Emergency surgery, mortality, total blood transfusion requirements, and hospitalisation after two endoscopic haemostatic treatments

\begin{tabular}{|c|c|c|}
\hline & Adrenaline & $\begin{array}{l}\text { Adrenaline } \\
\text { +laser }\end{array}$ \\
\hline Emergency surgery & $3 / 21(14 \%)$ & $\begin{array}{l}0 / 21 \\
0 / 21\end{array}$ \\
\hline $\begin{array}{l}\text { Mean (SD) total blood transfusion } \\
\text { requirement (units) }\end{array}$ & $5 \cdot 8(4 \cdot 3)$ & $5 \cdot 1(3 \cdot 2)$ \\
\hline Mean (SD) hospitalisation time (days) & $9 \cdot 9(7 \cdot 5)$ & $7 \cdot 8(3 \cdot 6)$ \\
\hline
\end{tabular}

gastric ulcer with a large non-bleeding visible vessel. Further adrenaline injections failed to control the bleeding and the patient required emergency surgery. A deep ulcer penetrating into the substance of the pancreas and eroding a large branch of the splenic artery was found and a high polya gastrectomy was performed. No treatment induced perforations or other complications occurred in either group.

\section{Discussion}

The patients included in the present comparison of two endoscopic treatments for peptic ulcer bleeding were at high risk of further haemorrhage and death. Several large studies have shown that rebleeding occurs in $40-60 \%$ of ulcers with a bleeding or non-bleeding visible vessel. .691112

The risk of rebleeding from a visible vessel is increased further by the presence of clinical shock and a haemoglobin $<80 \mathrm{~g} / \mathrm{l}$ on admission $^{13-15}$; these prognostic indicators were present in $52 \%$ and $29 \%$ of our patients respectively. Adrenaline injection treatment and combined adrenaline injection $+\mathrm{Nd}$ : $\mathrm{YAG}$ laser coagulation treatment achieved final haemostasis in $86 \%$ and $100 \%$ of these high risk patients respectively. As a result, only three patients in the adrenaline group required emergency surgery - an overall rate in the whole series of $7 \%$ - and more importantly none died as a consequence of peptic ulcer haemorrhage. The lack of deaths in this high risk subgroup assumes even greater importance if one remembers that in all patients with bleeding peptic ulcers - that is, with and without visible vessels - conservative management is associated with a mortality of around $10 \% .^{1617}$ The $86 \%$ final haemostasis with adrenaline $1: 10000$ injection in this study is similar to that reported in other series of patients with bleeding ulcers with visible vessels in which first rebleeds were also treated by repeat endoscopic treatment: Chung et al ${ }^{11} 86 \%$, Rutgeerts et al ${ }^{12} 83 \%$. In both of these trials adrenaline injection treatment caused a pronounced reduction in rebleeding compared with conservative management. The published experience with adrenaline preinjection +Nd:YAG laser coagulation for bleeding ulcers with visible vessels is likewise limited. In two different randomised studies Rutgeerts et $a l^{1218}$ reported $88 \%$ haemostasis after two sessions of combination treatment compared with $100 \%$ in the present study. Heldwen et $a l^{19}$ reported $78 \%$ haemostasis in a similar group of patients.

The excellent results of endoscopic haemostasis for peptic ulcer haemorrhage in the series by Chung et al, ${ }^{11}$ Rutgeerts et $a l,{ }^{12}$ and the present study highlight the importance of operator expertise. It is well recognised that there is a learning period, often up to six months, with all types of endoscopic haemostatic treatments. ${ }^{90}$ High levels of endoscopic skill can only be maintained if an operator has continued exposure to an adequate number of cases and is motivated to persist with what are often difficult and time consuming cases. Thus the results reported from centres with a special interest and expertise in endoscopic haemostasis and a 
large throughput of patients should not be expected in smaller units with less commitment to therapeutic endoscopy.

Animal experiments using actively bleeding mucosal ulcers have shown that submucosal injections of adrenaline $(1: 10000)$ secure haemostasis by arterial vasoconstriction and probably vessel compression by the injected fluid volume..$^{22}$ The mechanism of longterm haemostasis remains obscure. Adrenaline injections induce only a mild inflammatory response with no vasculitis or thrombosis of submucosal vessels. ${ }^{21}$ It may be that vessel compression by the injected volume of adrenaline allows enough time for retraction and partial fibrous organisation of the clot sealing the rent in the submucosal artery eroded by the ulcer. Because of the inability of submucosal adrenaline injections to produce vasculitis it has been proposed that additional treatment that produces vessel thrombosis either chemically (injections of sclerosing agents) or thermally (Heater or BICAP probe, $\mathrm{Nd}$ :YAG laser) may produce superior haemostasis rates. Although the efficacy of combination treatment in the present study (adrenaline+ $\mathrm{Nd}$ :YAG laser) and in that by Rutgeerts et $a l^{12}$ (adrenaline + polidocanol, adrenaline $+\mathrm{Nd}: \mathrm{YAG}$ laser) was numerically superior to that of adrenaline injection alone, the differences were not significant on statistical testing. Given the high efficacy of adrenaline injection any potential appreciable improvement with combination treatment could only be shown in a much larger trial.

In the present study submucosal injection of 1:10000 adrenaline into the stomach or duodenum, even in large volumes up to $30 \mathrm{ml}$, did not result in obvious cardiac arrhythmias or other systemic complications. This is due to the high clearance of any adrenaline reaching the liver via the portal venous system by the hepatic enzymes catechol-O-methyl transferase and monoamine oxidase. As patients were not routinely re-endoscoped in the first 48-72 hours after injection treatment, no comment can be made regarding the theoretical risk of ulcer extension as a result of local tissue ischaemia. In the study by Chung et al neither enlargement of the ulcers nor delay in healing was observed."

A randomised comparison of two endoscopic haemostatic modalities in a group of patients with bleeding peptic ulcers at high risk of further haemorrhage has shown that endoscopic treatment can secure haemostasis in the vast majority, obviating the need for emergency surgery. Both adrenaline injection and adrenaline injection+ $\mathrm{Nd}$ :YAG laser photocoagulation proved highly efficacious. Although the efficacy of combined treatment was numerically superior to that of injection alone, the difference was not significant $(0.05<\mathrm{p}<0.1)$. It is possible that this can be accounted for by a type II statistical error (insufficient patient numbers to show significance). Until this is proved in a trial of larger size adrenaline injection treatment seems to be the treatment of choice for most units in view of its simplicity, low cost, and wide availability. In endoscopy units that already have an Nd:YAG laser for other indications such as palliation of advanced upper and lower gastrointestinal cancers, combination treatment is a logical option in view of its potential small therapeutic advantage over adrenaline injection treatment alone. The policy of treating all first rebleeds with a second session of endoscopic treatment and reserving surgery for a second episode of rebleeding has proved safe and resulted in a higher overall endoscopic haemostasis rate with a concomitant reduction in emergency surgery.

This work has been supported by the Special Medical Development on Lasers of the Department of Health.

1 Dronfield MW. Medical or surgical management for haematemesis or melaena. $\mathcal{J} R$ Coll Physicians Lond 1979; 13: $84-6$.

2 Avery Jones F. Hematemesis and melaena with specia reference to factors influencing mortality from bleeding reference to factors influencing mortality fr

3 Clason AE, Macleod DAD, Elton RA. Clinical factors in the prediction of further haemorrhage or mortality in acute upper gastrointestinal haemorrhage. Br $\mathcal{F}$ Surg 1986; 73 985-7.

4 Foster DN, Miloszewski KJA, Losowsky MS. Stigmata of recent haemorrhage in diagnosis and prognosis of upper gastrointestinal bleeding. BMF 1978; 1: 1173-7.

5 Storey DW, Bown SG, Swain CP. Endoscopic prediction of recurrent bleeding in peptic ulcers. N Engl F Med 1981; 305 915-6.

6 Swain CP, Kirkham JS, Salmon PR, Bown SG, Northfield TC. Controlled trial of Nd:YAG laser photocoagulation in bleeding peptic ulcers. Lancer 1986; i: 1113-6.

7 MacLeod IA, Mills AR, Mackenzie JF, et al. Nd:YAG laser photocoagulation for major haemorrhage from peptic ulcers and single vessels. A single blind controlled study. $B M \mathcal{F}$ 1983; 286: 345-8

8 Trudeau W, Siepler JK, Russ K, et al. Endoscopic Nd: YAG laser photocoagulation of bleeding ulcers with visible vessels. Gastrointest Endosc 1985; 31: 138-41

9 Matthewson K, Swain CP, Bland M, Kirkham JS, Bown SG, Northfield TC. A randomized comparison of Nd:YAG laser, heater probe and no endoscopic therapy for bleeding peptic ulcer. Gastroenterology 1990; 98: 1239-44.

10 Rutgeerts P, Vantrappen G, Broeckaert L, Coremans G, Janssens J, Geboes K. A new and effective technique of YAG laser photocoagulation for severe upper gastrointestinal bleeding. Endoscopy 1984; 16: 115-7.

11 Chung SCS, Leung JWC, Steele RJC, Crofts TJ, Li AKC. Endoscopic injection of adrenaline for actively bleeding ulcers: a randomised trial. $B M \mathcal{F} 1989 ; 296: 1631-3$.

12 Rutgeerts P, Vantrappen G, Broeckaert L, Coremans G, Janssens J, Hiele M. Comparison of endoscopic polidocanol injection and YAG laser therapy for bleeding peptic ulcers. Lancet 1989; i: $1164-6$.

13 Bornman PC, Theodorou NA, Shuttleworth D, Essel HP, Marks IN. Importance of hypovolaemic shock and endoscopic signs of predicting recurrent haemorrhage from peptic ulcer: a prospective evaluation. $B M F$ 1985; 291: peptic $245-7$.

14 Hunt PS. Bleeding gastroduodenal ulcers: selection of patients for surgery. World $\mathcal{F}$ Surg 1987; 11: 289-94.

15 Clason AE, Macleod DAD, Elton RA. Clinical factors in the predication of further haemorrhage in acute upper gastrointestinal haemorrhage. BrF Surg 1986; 73: 985-7.

16 Schiller KFR, Truelove SC, Williams GD, Gwyn D. Haematemesis and melaena with special reference to factors influencing outcome. $B M \mathcal{F}$ 1970; ii: 7-14.

17 Vellacot KD, Dronfield MW, Langman MJS. Comparison of medical and surgical management of bleeding peptic ulcers. BMF 1982; i: 548-50.

18 Rutgeerts P, Vantrappen G, Van Hootegem Ph, et al. Nd:YAG laser photocoagulation versus multipolar electrocoagulation for the treatment of severely bleeding ulcers: a randomized for the treatment of severely bleeding ulcers: a rand

19 Heldwein W, Lehnert P, Martinoff S, Loeschka K. Local epinephrine injection improves the therapeutic effect of epinephrine injection improves the therapeutic effect of
Nd:YAG laser treatment of arterial peptic ulcer bleeding. Endoscopy 1988; 20: 2-4.

20 O'Brien JD, Day SJ, Burnham WR. Controlled trial of smal bipolar probe in bleeding peptic ulcers. Lancet 1986; $\mathrm{i}$ 464-6.

21 Chung SCS, Leung JWC, Galvina M, Lee TW. The effect of submucosal adrenaline on blood loss from standard bleeding ulcers. Gut 1987; 28: A1402.

22 Rutgeerts P, Geboes K, Vantrappen G. Experimental studies of injection therapy for severe non-variceal bleeding in dogs. Gastroenterology 1989; 97: 610-21. 